\title{
Nasal colonization and antimicrobial susceptibility pattern of Staphylococcus aureus among pre-school children in Ethiopia
}

Alemayehu Reta ${ }^{1 *}$, Moges Wubie $^{2}$ and Getnet Mekuria²

\begin{abstract}
Background: Staphylococcus aureus is one of the bacterium that can asymptomatically colonize the human upper respiratory tract (i.e. nose and throat). Carriage of $S$. aureus, including methicillin resistant $S$. aureus, is common to children. The aim at this study was to determine the nasal colonization, associated factors and antimicrobial susceptibility patterns of S. aureus isolates among pre-school children in Debre Markos town.

Methods: Institutional-based cross sectional study was conducted. A total of 400 nasal swabs were collected from pre-school children from April to June, 2015 following standard microbiological methods. MRSA was detected using both Cefoxitin $(30 \mu \mathrm{g})$ and Oxacillin $(6 \mu \mathrm{g})$ (Oxoid Ltd. England) discs in combination and associated factors were assessed using self-administered pretested questionnaires, which were delivered to the children's parents/guardians. Statistical analysis of the data (logistic regression) was done using SPSS V-22.

Results: A total of $52 \mathrm{~S}$. aureus isolate was recovered from 400 nasal swap samples. The prevalence of $\mathrm{S}$. aureus among pre-School children was 13\% (52/400). The susceptibility patterns of the isolates to commonly used antibiotics were: $84.62 \%$ to Chloramphenicol, $69.2 \%$ to Doxycycline and Tetracycline, $92.3 \%$ to Kanamycin, $7.7 \%$ to Ampicillin and Penicillin, $86.6 \%$ to Ceftriaxone, and $76.9 \%$ to Augmentin. All the isolates were sensitive to Oxacillin and Cefoxitin, mean there was no methicillin resistant $S$. aureus isolate, and also sensitive to Gentamycin, Erythromycin and Clindamycin. The main associated factors of nasal colonization of $S$. aureus in the study area was, having recurrent acute otitis media $(A O M)[A O R=2.37(1.11,5.06)]$, Children admission in hospital $[A O R=1.96(1.03,3.73)]$ and cough $[\mathrm{AOR}=2.09(1.08,4.09)]$.

Conclusions: The prevalence of $S$. aureus nasal colonization among pre-school children was relatively low in absence of MRSA. Factors like; recurrent AOM, hospital admission and cough were significantly associated with S. aureus nasal colonization. Most of the isolates were resistant to $\beta$-lactam drugs and sensitive to drugs like Gentamycin, Erythromycin, Clindamycin, Chloramphenicol, Doxycycline, Tetracycline, Kanamycin and Augmentin.
\end{abstract}

Keywords: Antimicrobial susceptibility pattern, Staphylococcus aureus, Nasal colonization, Pre-school children

\section{Background}

Nasal colonization of $S$. aureus creates a risk factor of developing invasive disease. Children are commonly considered to be persistent carriers more frequently than adults, with the highest rate of carriage reached in the first year of life and transition from persistent to

\footnotetext{
${ }^{*}$ Correspondence: alemayehureta@yahoo.com

1 Department of Medical Laboratory Science, College of Health Sciences, Debre Markos University, Pobox-269, Debre Markos, Ethiopia Full list of author information is available at the end of the article
}

intermittent or non-carriage occurring during adolescence [1].

There is increasing evidence that $S$. aureus nasal colonization is spreading among healthy pre-school children. Studies conducted around the world reveal that, $17.5 \%$ in Japan [2], 34\% in Belgium [3], 35\% in India [4] and Republic of Korea [5], 48\% in Brazil [6], and 17.3\% in Turkey [7] of pre-school children were colonized by S. aureus, even if the associated factors are not readily identified. While some studies have demonstrated recurrent acute 
otitis media (AOM), frequent exposure to antibiotics [8], socioeconomic status [9], healthcare worker in the home [10], child-care attendance [11], hospitalization, chronic illness and previous isolation of methicillin resistant $S$. aureus (MRSA) [12] but there is no definite consensus as to why healthy children acquire these resistant bacteria [9]. There are few studies conducted to assess nasal colonization of $S$. aureus among pre-school children around the globe and to our best knowledge, there is no study conducted on factors associated with nasal colonization of S. aureus particularly among pre-school children in the study area and in Ethiopia at large. Therefore this study will create a better understanding of the epidemiology and determinants of $S$. aureus nasal colonization could positively be helpful in improving infection control and prevention strategies and designing protocols.

\section{Methods}

\section{Study enrollment, specimen collection and processing}

Institution based cross sectional study was conducted at kindergarten school from April to June, 2015. All sampled pre-school children (1-6 years) who were attending their kindergarten education in Debre Markos town during the study period were selected by using simple random sampling technique. Children were excluded from the study, if they had received antibiotic treatment for the previous 7 days. Individual variables were obtained from the children's guardians/parents immediately after written consent is obtained from the guardians/parents and before swabs were collected. After written informed consent was obtained, each child had a specimen collected from the anterior nares with a dry, sterile and moistened swab (Ventura Tran system; Copan Diagnostics, Corona, Calif.). The tip of the collection swab was inserted $2-3 \mathrm{~cm}$ into the anterior nares and rotated 4-5 times both clockwise and anticlockwise before withdrawal in each nostril. Swabs were placed into Screw capped test tubes containing enrichment broth (contained 37.5 g sodium chloride $(\mathrm{NaCl}), 1.25 \mathrm{~g}$ yeast extract, $5.0 \mathrm{~g}$ tryptone, and $500 \mathrm{ml}$ distilled water) and the samples were labeled, packaged and transported to the Medical Microbiology Laboratory of Debre Markos University within $2 \mathrm{~h}$ of collection and processed immediately according to standard Microbiological procedures [13].

\section{Selective media and culture conditions}

Each $10 \mu \mathrm{l}$ of incubated enrichment broth was inoculated in both blood agar and MSA (Oxoid Ltd., England) and incubated at $35{ }^{\circ} \mathrm{C}$ for $24-48 \mathrm{~h}$. Golden yellow colonies of the MSA and white creamy colonies in blood agar indicate $S$. aureus, which was subsequently identified by gram staining, $3 \%$ catalase testing, tube coagulase testing with the Staph Latex agglutination assay (Life Sign, Somerset, N.J.) [14].

\section{Identification of MRSA}

Staphylococcus aureus isolates were tested for Methicillin resistance by using modified Kirby-Bauer disc diffusion technique [15]. Inocula were adjusted to $0.5 \%$ McFarland standard and each streaked uniformly with the swab sticks in Mueller-Hinton agar plates containing $6.5 \% \mathrm{NaCl}$ to obtain confluent growth [16]. The plates were allowed to dry for 3-5 min before the disks used. The Oxacillin $(6 \mu \mathrm{g})$ and Cefoxitin $(30 \mu \mathrm{g})$ discs were used for susceptibility testing. The plates then incubated aerobically at $35^{\circ} \mathrm{C}$ for $24 \mathrm{~h}$. The results were interpreted according to Clinical and Laboratory Standard Institute (CLSI) guidelines for Oxacillin and Cefoxitin susceptibility testing: a zone size of $<10 \mathrm{~mm}$ was considered resistant; a zone size of $>13 \mathrm{~mm}$ was considered susceptible for Oxacillin and a zone size of $<21 \mathrm{~mm}$ was considered resistant; a zone size of $>22 \mathrm{~mm}$ was considered susceptible for Cefoxitin [17].

\section{Antibiotic susceptibility testing}

The antibiotic susceptibility testing was performed by modified Kirby-Bauer disc diffusion technique and MIC for Ceftriaxone [15]. The colonies were picked up with wooden applicator stick and dipped into nutrient broth to make direct colony suspension of the isolates and inocula were adjusted at $0.5 \%$ McFarland standard. After few minutes these suspensions were streaked with Mueller-Hinton agar plates. The antibiotic susceptibility testing was performed on the following antibiotic discs; Ceftriaxone $(30 \mu \mathrm{g})$, Chloramphenicol $(30 \mu \mathrm{g})$, Clindamycin $(2 \mu \mathrm{g})$, Erythromycin $(15 \mu \mathrm{g})$, Gentamicin $(10 \mu \mathrm{g})$, Penicillin G (10 units), Doxycycline (30 $\mu \mathrm{g})$, Tetracycline $(30 \mu \mathrm{g})$, Kanamycin $(30 \mu \mathrm{g})$, Amoxicillin-Clavulanic acid $(20 \mu \mathrm{g})$, Cefoxitin $(30 \mu \mathrm{g})$, Ampicillin $(10 \mu \mathrm{g})$ and Oxacillin $(6 \mu \mathrm{g})$. All antibiotic discs/drugs were obtained from Oxoid Ltd. England. The plates were incubated at $37{ }^{\circ} \mathrm{C}$ for $18-24 \mathrm{~h}$.

The disc diffusion technique and zone interpretation of each antimicrobial agent were used in accordance with CLSI guidelines [16]. Diameters of the zone of inhibition around the disc were measured using a graduated caliper in millimeters and the isolates was classified as sensitive, intermediate, and resistant according to the standardized table supplied by the CLSI. Staphylococcus aureus (ATCC-25923) was used as control for the antimicrobial susceptibility pattern.

\section{Data analysis}

The collected data were checked for completeness, coded, and then entered into Epi data version 3.1 software for an 
edition and cleaning of data and export to SPSS version 22 Software for analysis. Bivariate and multivariate logistic regression analysis were performed to identify factors associated with nasal colonization of $S$. aureus.

\section{Results}

Socio-demographic characteristics

Of the 422 eligible parents/guardians, 400 agreed to participate in the study, which made a response rate of $94.8 \%$. The median age of pre-school children were 5 years (Inter quartile range, IQR \pm 3 ). With regard to household monthly income, the median income were 3100 birr (Inter quartile range, IQR $\pm 16,267$ ). More than half (55\%) of households had income of 2001-5000 birr monthly (Table 1).

\section{Prevalence of S. aureus}

About 74\% (296/400) of the pre-school children involved in the study were aged $>4$ years, of these $12 \%(48 / 400)$ were colonized by S. aureus. Majority of the study participants were females, which accounts 60\% (240/400) among them $8 \%(32 / 400)$ were colonized by $S$. aureus. The overall prevalence of $S$. aureus were 13\% (52/400) (Table 2). The good news from our study was, there was

Table 1 Socio-demographic characteristics of pre-school children, Debre Markos, North Ethiopia, 2015

\begin{tabular}{lc}
\hline Demographic characteristics $(\mathbf{n}=\mathbf{4 0 0})$ & No (\%) \\
\hline Sex of child & \\
Male & $160(40.0)$ \\
Female & $240(60.0)$ \\
Age of child (years) & \\
$\leq 4$ & $169(42.3)$ \\
$>4$ & $231(57.8)$ \\
Younger sibling & \\
0 & $240(60.0)$ \\
$1-3$ & $160(40.0)$ \\
Older sibling & \\
0 & $136(34.0)$ \\
1-3 & $264(66.0)$ \\
Educational status of mother & \\
Unable to read \& write & $32(8.0)$ \\
Primary education & $72(18.0)$ \\
Secondary education & $152(38.0)$ \\
College \& above & $144(36.0)$ \\
Household monthly income & \\
$400-2000$ & $112(28.0)$ \\
$2001-5000$ & $220(55.0)$ \\
$5001-16,667$ & $68(17.0)$ \\
\hline a Ethiopian Birr &
\end{tabular}

a Ethiopian Birr
Table 2 Age and sex distribution of S. aureus colonization among pre-school children Debre Markos town, 2015

\begin{tabular}{llll}
\hline Characteristics & \multicolumn{2}{l}{ S. aureus colonization } & Total \\
\cline { 2 - 3 } & Yes & No & \\
\hline Age & & & \\
Median & 6 & 5 & 5 \\
Range & 3 & 3 & 3 \\
Age (years) & & & \\
$>4$ & $48(12 \%)$ & $248(62 \%)$ & $296(74 \%)$ \\
$\leq 4$ & $4(1 \%)$ & $100(25 \%)$ & $104(26 \%)$ \\
Total & $52(13 \%)$ & $348(87 \%)$ & $400(100 \%)$ \\
Sex & & & \\
Male & $20(5 \%)$ & $140(35 \%)$ & $160(40 \%)$ \\
Female & $32(8 \%)$ & $208(52 \%)$ & $240(60 \%)$ \\
Total & $52(13 \%)$ & $348(87 \%)$ & $400(100 \%)$ \\
\hline
\end{tabular}

no MRSA isolates among S. aureus colonized pre-school children.

\section{Medical and related characteristics}

The majority (87.8\%) of pre- school children didn't colonize by S. aureus. Fifty-seven (14.3\%) of pre-school children had recurrent AOM. More than half $(52.8 \%)$ of pre-school children had Cough in the previous 30 days. One-fourth (25.3\%) of pre-school children was admitted to hospital in the previous 12 months (Table 3 ).

\section{Factors associated with nasal colonization of $S$. aureus}

Hosmer-Lemeshow goodness-of-fit tested $(\mathrm{p}=0.425)$ was used to assess the fitness of the model. During the

Table 3 Medical and related characteristics of pre-school children, Debre Markos, North Ethiopia, 2015

\begin{tabular}{|c|c|}
\hline Characteristics & No $(\%)(n=400)$ \\
\hline Presence of S. aureus (child) & $49(12.3)$ \\
\hline Skin/soft tissue infection (child) & $107(26.8)$ \\
\hline Having recurrent acute otitis media (child) & $57(14.3)$ \\
\hline $\operatorname{Cough}^{\mathrm{a}}($ child $)$ & $211(52.8)$ \\
\hline Child admitted to hospital ${ }^{b}$ & $101(25.3)$ \\
\hline Child use of antibiotic $^{b}$ & $201(50.3)$ \\
\hline Use of antibiotic by a child of the household ${ }^{b}$ & $197(49.3)$ \\
\hline Use of antibiotic by a family member ${ }^{b}$ & $180(45.0)$ \\
\hline Hospitalization of family member ${ }^{b}$ & $52(13.0)$ \\
\hline $\begin{array}{l}\text { Household member contact with health institution } \\
\text { workers }\end{array}$ & $120(30.0)$ \\
\hline Family member with wound ${ }^{b}$ & $94(23.5)$ \\
\hline
\end{tabular}


bivariate logistic regression analysis, child admission to hospital in the previous 12 months, Skin/soft tissue infection, having recurrent $\mathrm{AOM}$, cough in the previous 30 days, family member with wound in the previous 12 months and child use of antibiotic in the previous 12 months were significantly associated with nasal colonization of $S$. aureus.

During the multivariate logistic regression analysis, child admission to hospital in the previous 12 months, having recurrent $\mathrm{AOM}$ and cough in the previous 30 days was significantly associated with nasal colonization of $S$. aureus.

Children who were admitted in hospital in the previous 12 months were 1.96 times more likely colonized by S. aureus than those children not admitted $[\mathrm{AOR}=1.96$ $(1.03,3.73)]$. Those children that had recurrent AOM were 2.37 times more likely colonized by $S$. aureus than children hadn't recurrent AOM [AOR $=2.37(1.11,5.06)]$. Those children that had coughed in the previous 30 days were 2.09 times more likely colonized by $S$. aureus than children hadn't coughed [AOR $=2.09(1.08,4.09)]$ (Table 4).

\section{Evaluation of antimicrobial susceptibility tests}

The in vitro antimicrobial susceptibility pattern results in $52 \mathrm{~S}$. aureus isolates were shown in Table 2. A total of $52 \mathrm{~S}$. aureus isolates were subjected to antibiotic susceptibility test against 13 antimicrobial drugs (Table 5). The susceptibility patterns of the isolates to commonly used antibiotics were: $84.62 \%$ to Chloramphenicol, $69.2 \%$ to Doxycycline and Tetracycline, $92.3 \%$ to Kanamycin, $7.7 \%$ to Ampicillin and Penicillin, $86.6 \%$

Table 5 Antimicrobial susceptibility patterns of $S$. aureus isolates, Debre Markos town, 2015

\begin{tabular}{llll}
\hline $\begin{array}{l}\text { Antimicrobial } \\
\text { drugs }\end{array}$ & \multicolumn{3}{l}{ S. aureus isolates susceptibility pattern, $\mathbf{N}=\mathbf{5 2}$} \\
\cline { 2 - 4 } & $\begin{array}{l}\text { Sensitive no } \\
\text { (\%) }\end{array}$ & $\begin{array}{l}\text { Intermediate } \\
\text { no (\%) }\end{array}$ & Resistant no (\%) \\
\hline Penicillin & $4(7.7)$ & $0(0)$ & $48(92.3)$ \\
Ampicillin & $4(7.7)$ & $0(0)$ & $48(92.3)$ \\
Oxacillin & $52(100)$ & $0(0)$ & $0(0)$ \\
Cefoxitin & $52(100)$ & $0(0)$ & $0(0)$ \\
Augmentin & $40(76.9)$ & $0(0)$ & $12(23.1)$ \\
Ceftriaxone & $52(100)$ & $0(0)$ & $0(0)$ \\
Gentamycin & $52(100)$ & $0(0)$ & $0(0)$ \\
Kanamycin & $48(92.3)$ & $4(7.7)$ & $0(0)$ \\
Erythromycin & $52(100)$ & $0(0)$ & $0(0)$ \\
Tetracycline & $36(69.2)$ & $0(0)$ & $16(30.8)$ \\
Doxycycline & $36(69.2)$ & $4(7.7)$ & $12(23.1)$ \\
Clindamycin & $52(100)$ & $0(0)$ & $0(0)$ \\
Chlorampheni- & $44(84.62)$ & $0(0)$ & $8(15.38)$ \\
col & & & \\
\hline
\end{tabular}

Table 4 Bivariate and multivariate logistic regression analysis showing factors associated with nasal colonization by S. aureus among pre-school children, Debre Markos, North Ethiopia, 2015

\begin{tabular}{|c|c|c|c|c|c|}
\hline \multirow[t]{2}{*}{ Variable } & \multicolumn{2}{|c|}{ Presence of S. aureus } & \multirow[t]{2}{*}{ Crude odds ratio $(95 \% \mathrm{Cl})$} & \multirow[t]{2}{*}{ Adjusted odds ratio $(95 \% \mathrm{Cl})$} & \multirow[t]{2}{*}{$P$ value } \\
\hline & No (\%) & Yes (\%) & & & \\
\hline \multicolumn{6}{|c|}{ Child admitted to hospital in the previous 12 months } \\
\hline Yes & $20(5.0)$ & $81(20.2)$ & $2.29(1.24,4.28)$ & $1.96(1.03,3.73)$ & 0.040 \\
\hline No & $29(7.2)$ & $270(67.5)$ & 1 & 1 & \\
\hline \multicolumn{6}{|c|}{ Skin/soft tissue infection } \\
\hline Yes & $19(4.8)$ & $88(22.0)$ & $1.89(1.02,3.53)$ & & \\
\hline No & $30(7.5)$ & $263(65.8)$ & 1 & & \\
\hline \multicolumn{6}{|c|}{ Having recurrent $\mathrm{AOM}$} \\
\hline Yes & $12(3.0)$ & $45(11.2)$ & $2.21(1.07,4.54)$ & $2.37(1.11,5.06)$ & 0.026 \\
\hline No & $37(9.2)$ & $306(76.5)$ & 1 & 1 & \\
\hline \multicolumn{6}{|c|}{ Cough in the previous 30 days } \\
\hline Yes & $34(8.5)$ & $177(44.2)$ & $2.23(1.17,4.24)$ & $2.09(1.08,4.09)$ & 0.029 \\
\hline No & $15(3.8)$ & $174(43.5)$ & 1 & 1 & \\
\hline \multicolumn{6}{|c|}{ Family member with wound in the previous 12 months } \\
\hline Yes & $18(4.5)$ & $76(19.0)$ & $2.10(1.12,3.96)$ & & \\
\hline No & $31(7.8)$ & $275(68.8)$ & 1 & & \\
\hline \multicolumn{6}{|c|}{ Child use of antibiotic in the previous 12 months } \\
\hline Yes & $18(4.5)$ & $183(45.8)$ & $1.88(1.01,3.48)$ & & \\
\hline No & $31(7.8)$ & $168(42.0)$ & 1 & & \\
\hline
\end{tabular}

Backward stepwise logistic regression 
to Ceftriaxone (but after performing minimum inhibitory concentration (MIC), all the isolates become susceptible for Ceftriaxone), and $76.9 \%$ to Augmentin. All the isolates were sensitive to Oxacillin and Cefoxitin, mean there was no MRSA isolate and also sensitive to Gentamycin, Erythromycin and Clindamycin.

\section{Drug resistance pattern/antibiogram of S. aureus}

The resistance patterns of $S$. aureus isolates varied from one to six antimicrobial drugs. The highest resistance pattern (46.15\%) was observed for two antibiotics with pattern of Penicillin/Ampicillin. Three resistance patterns of equal proportion were observed for resistance to one, three and six antibiotics such as Chloramphenicol, Penicillin/Ampicillin/Tetracycline and Penicillin/ Ampicillin/Augmentin/Tetracycline/Doxycycline/Chloramphenicol, respectively and also Penicillin/Ampicillin/ Augmentin and Penicillin/Ampicillin/Tetracycline/Doxycycline had the same resistance pattern proportion. Maximum resistance (resistant to six drugs) was observed for one $S$. aureus isolate from resistance pattern of Penicillin/ Ampicillin/Augmentin/Tetracycline/Doxycycline/Chloramphenicol. Multi-drug resistance (defined as resistance to at least three different antibiotic groups) S. aureus isolates were observed (Table 6).

\section{Discussion}

In the present study, the overall frequency of isolation of $S$. aureus from pre-school children of Debre Markos town was $13 \%(52 / 400)$. In contrast with this, a study conducted in Belgium, [3], around 50\% of pre-school children were colonized with $S$. aureus. This study also performed a prospective cohort study, how socio-economic status affects $S$. aureus nasal colonization among preschool children, and conclude that there is high $S$. aureus nasal colonization among preschool children that have high socio-economic status when we compare with low socio-economic status. As we know our

Table 6 Drug resistance pattern of $S$. aureus isolates for different antibiotics, Debre Markos town, 2015

\begin{tabular}{llc}
\hline $\begin{array}{l}\text { No of antimicrobial } \\
\text { drugs }\end{array}$ & Resistance pattern & $\begin{array}{l}\text { Resistant isolates, } \\
\text { No (\%) }\end{array}$ \\
\hline 1 & Chl & $4(7.69)$ \\
2 & Pen/Amp & $24(46.15)$ \\
3 & Pen/Amp/Aug & $8(15.38)$ \\
& Pen/Amp/Tet & $4(7.69)$ \\
4 & Pen/Amp/Tet/Dox & $8(15.38)$ \\
6 & Pen/Amp/Aug/Tet/ & $4(7.69)$ \\
& Dox/Chl & \\
\hline
\end{tabular}

Pen penicillin, Amp ampicillin, Aug augmentin, Tet tetracycline, Dox doxycycline, Chl chloramphenicol country, Ethiopia is one of a developing country having low socio-economic status. This factor may be the reason why the current study obtained decreased $S$. aureus nasal colonization among pre-school children in Debre Markos town. In addition to this, seasonal variations, geographical distribution and method of isolation may affect the prevalence.

In line with the current study, a study conducted in Japan, [2], the prevalence of $S$. aureus nasal colonization among children attending day care center having 1 month-5 year ages was $17.9 \%$ and also a study conducted in Turkey, [7] reveals that, $17.3 \%$ of pre-school children were colonized by $S$. aureus.

The susceptibility patterns of the isolates to commonly used antibiotics were: $84.62 \%$ to Chloramphenicol, $69.2 \%$ to Doxycycline and Tetracycline, $92.3 \%$ to Kanamycin, 7.7\% to Ampicillin and Penicillin, 86.6\% to Ceftriaxone, and $76.9 \%$ to Augmentin. All the isolates were sensitive to Oxacillin, Gentamycin, Erythromycin and Clindamycin. The findings of our current study were similar with a study conducted in Turkey [7], all S. aureus isolates were sensitive to Gentamycin, Erythromycin and Clindamycin.

Our finding showed that, there is no methicillin resistant $S$. aureus isolates. This finding is in agreement with a study conducted in Turkey, [7], they isolated only one MRSA (0.1\%) from a newborn infant that had been hospitalized during the first week of his life in the neonatal intensive care unit, which may suggest hospital acquired/ nosocomial origin.

The prevalence of carriage and the effect of various epidemiological factors of nasal colonization of $S$. aureus has been described, but results vary from one study to another, in parallel with differences in the populations included or definitions used.

A study conducted in India shows that, attending kindergarten schools or being in pre-school are one factor to be colonized with S. aureus [4]. Through such investigation these pre-school children could be target groups to undergo further assessment on factors related to nasal colonization of pre-school children with $S$. aureus. In our current study, the main associated factors of nasal colonization of $S$. aureus among pre-school children was Child admitted to hospital in the previous 12 months [AOR $=1.96$ (1.03, 3.73); $\mathrm{p}=0.040]$, having recurrent $\mathrm{AOM}[\mathrm{AOR}=2.37(1.11,5.06) ; \mathrm{p}=0.026]$ and Cough in the previous 30 days $[\mathrm{AOR}=2.09(1.08,4.09) ; \mathrm{p}=0.029$ ].

A study conducted among North Carolina children states that, antibiotic use by children in the past 6 months were associated with nasal colonization of $S$. aureus. In addition to that, the study also reveals that, parental employment in a school/daycare and family history of boils were associated with $S$. aureus nasal carriage [9]. In line with that, our study also obtained family member 
with wound in the previous 12 months were associated with nasal colonization of $S$. aureus in the bivariate logistic regression analysis.

Concerning about recurrent AOM in the previous 12 months, a study conducted in Ethiopia by the corresponding author of this research (Alemayehu Reta) shows statistically significant association among school children [8].

In agreement with our finding, a study conducted in Nepal by Rijal KR and his colleagues illustrates that, children admitted to hospital in the past 12 months were statistically associated with nasal colonization of $S$. aureus [12] and also a study in Turkey children shows a similar finding, which is conducted among children having the age of 6 months up to 15 years, hospitalization or surgical operation in the previous 1 year were associated with S. aureus colonization [18] obviously, there are infectious agents in the hospital environment that can be considered as hospital acquired infection. S. aureus is one of the nosocomial infectious agent that can disseminate to the community when an admitted patient is discharged. So if the child is admitted previously, there will be a greater probability of being colonized by $S$. aureus.

\section{Conclusions and recommendations}

This is the first study among Ethiopian pre-school children below 6 years of age studying nasal colonization of $S$. aureus and antimicrobial susceptibility pattern of the isolates and showed a relatively low prevalence of $S$. aureus. Most of the isolates were resistant to $\beta$-lactam drugs and sensitive to drugs like Gentamycin, Erythromycin, Clindamycin, Chloramphenicol, Doxycycline, Tetracycline, Kanamycin and Augmentin. The study shows that attending pre-school was associated with nasal colonization of $S$. aureus. Studies with cohort design plus molecular analysis of the isolates are needed to accurately assess the epidemiology of $S$. aureus nasal colonization in various geographical locations, to see the strain distribution and to detect heteroresistant isolates. More studies on additional associated factors with a large sample size, which accompanies various geographical locations should be done for a better understanding of $S$. aureus nasal colonization and to design preventive strategies that target bacterial colonization in the upper respiratory tract (throat, nose...).

\footnotetext{
Abbreviations

AOM: acute otitis media; AOR: adjusted odds ratio; ATCC: American Type Culture Collection; Cl: confidence interval; CLSI: Clinical and Laboratory Standard Institute; IQR: inter quartile range; MIC: minimum inhibitory concentration; MRSA: methicillin resistant Staphylococcus aureus; MSA: mannitol salt agar; SPSS: Statistical Package for the Social Science.
}

Authors' contributions

AR carried out bacteriological studies, participated in the Microbiological analysis of samples, interpretation of data, drafted the manuscript, revising it critically for important intellectual content, given final approval of the version to be published. GM participated in acquisition of funding, collection of data and statistical analysis. MU participated in acquisition of funding and general supervision of the research group. All authors read and approved the final manuscript.

\section{Authors' information}

AR Medical Microbiologist, department head of Medical Laboratory Science, reviewer, researcher, give community service, advisor ship, and academic staff. GM Human Nutritionist, MU Infectious Disease, all perform activities like: research, give community service, advisor ship, and academic staff.

\section{Author details}

${ }^{1}$ Department of Medical Laboratory Science, College of Health Sciences, Debre Markos University, Pobox-269, Debre Markos, Ethiopia. ${ }^{2}$ Department of Public Health, College of Health Sciences, Debre Markos University, Debre Markos, Ethiopia.

\section{Acknowledgements}

We would like to acknowledge Debre Markos University for funding, Molla Yigzaw for sample collection and Getiye Dejenu for his contribution during data analysis.

\section{Competing interests}

The authors declare that they have no competing interests.

\section{Availability of data and materials}

The datasets generated and/or analyzed during the current study are not publicly available due a restriction by the University research legislation and some ethical issue but are available from the corresponding author on reasonable request.

\section{Consent to publish}

Not applicable.

\section{Ethics approval and consent to participate}

Ethical clearance was obtained from Debre Markos University ethical review board. Letter of permission to conduct the study was obtained from the director of each school. Informed consent was obtained from each study participant's guardian/parent. The purpose of the study was clearly described to the study participants including the benefits and risks of the study. Any information about the participants was kept confidential and the specimen collected from the participants was only analyzed for the intended purposes. The participants were enrolled only after they are sufficiently counseled and their informed consents have been obtained. Relevant confidentiality was maintained for every participant. After analysis of the participant's specimen, the positive findings for $S$. aureus were decolonized by using $2 \%$ Muprocin.

\section{Funding}

The research was funded by Debre Markos University and we declare that the funder do not participate in the design of the study and collection, analysis, and interpretation of data and in writing the manuscript.

\section{Publisher's Note}

Springer Nature remains neutral with regard to jurisdictional claims in published maps and institutional affiliations.

Received: 17 July 2016 Accepted: 12 December 2017

Published online: 19 December 2017

References

1. Wertheim HFL, Melles DC, Vos MC, Leeuwen W, Belkum A, Verbrugh HA, et al. The role of nasal carriage in Staphylococcus aureus infections. Lancet Infect Dis. 2005;5:751-62. 
2. Masuda K, Masuda R, Nishi J, Tokuda K, Yoshinaga M, Miyata K. Incidences of nasopharyngeal colonization of respiratory bacterial pathogens in Japanese children attending day-care centers. Pediatr Int. 2002;44:376-80.

3. Jourdain S, Smeesters P, Denis O, Dramaix M, Sputael V, Malaviolle X, et al. Differences in nasopharyngeal bacterial carriage in preschool children from different socio-economic origins. Clin Microbiol Infect. 2011;17:907-14.

4. Pathak A, Yogyata M, lyer RV, Singh B, Sharma M, Bo Eriksson, et al. Nasal carriage and antimicrobial susceptibility of Staphylococcus aureus in healthy preschool children in Ujjain, India. BMC Pediatr. 2010;10:100.

5. Bae S, Yu JY, Lee K, Lee S, Park B, Kang Y. Nasal colonization by four potential respiratory bacteria in healthy children attending kindergarten or elementary school in Seoul, Korea. J Med Microbiol. 2012;61:678-85.

6. Lamaro CJ, de Lencastre H, Kipnis A, Pimenta FC, Oliveira Luciana SC, Oliveira Renato M, et al. Molecular epidemiology and risk factors for nasal carriage of Staphylococcus aureus and methicillin-resistant S. aureus in infants attending day care centers in Brazil. J Clin Microbiol. 2009:47(12):3991-7.

7. Soysal A, Hulya S, Yegci A, Barlan I, Bakir M. The low rate of methicillinresistant Staphylococcus aureus in Turkish children. Jpn J Infect Dis. 2006:59:195-6.

8. Reta A, Gedefaw L, Sewunet T, Beyene G. Nasal carriage, risk factors and antimicrobial susceptibility pattern of methicillin resistant Staphylococcus aureus among school children in Ethiopia. J Med Microb Diagn. 2015;4:177. https://doi.org/10.4172/21610703.1000177.

9. Shapiro A, Raman S, Johnson M, Piehl M. Community-acquired MRSA infections in North Carolina children. NC Med J. 2009;70(2):102-7.

10. Woods SE, Beiter E, Drake B, Engel A. The prevalence of asymptomatic methicillin-resistant Staphylococcus aureus in school-age children. E J Med. 2011;16:18-21.
11. Dietrich DW, Auld DB, Mermel LA. Community-acquired methicillin-resistant Staphylococcus aureus in southern New England children. Pediatrics. 2004;113(4):e347.

12. Rijal KR, Pahari N, Shrestha BK, Nepal AK, Paudel B, Mahato P, et al. Prevalence of methicillin resistant Staphylococcus aureus in school children of Pokhara. Nepal Med Coll J. 2008;10(3):192-5.

13. Brown DFJ, Hawkey PM, Edwards DI, Morrison D, Ridgway GL, Towner KJ, et al. Guidelines for the laboratory diagnosis and susceptibility testing of methicillin-resistant Staphylococcus aureus (MRSA). J Antimicrob Chemother. 2005;56:1000-18

14. Kaleem F, Usman J, Hassan A, Omair M, Khalid A, Roz U. Sensitivity pattern of methicillin resistant Staphylococcus aureus isolated from patients admitted in a tertiary care hospital of Pakistan. Iran J Microbiol. 2010;2(3):141-3.

15. Cheesbrough M. District laboratory practice in tropical countries, vol. II. England: Cambridge University Press; 2002. p. 225-48.

16. Clinical and laboratory standards institute. Performance standards for antimicrobial disc susceptibility tests. Twelfth international supplement. 2011; M100-S20.

17. Mcdougal KL, Thornsberry C. New recommendations for disk diffusion antimicrobial susceptibility tests for methicillin-resistant (heteroresistant) staphylococci. J Clin Microbiol. 1984;19(4):482-8.

18. Ozgüven $A$, Tünger $O$, Cetin CB, Dinç $G$. Investigation of nasal carriage of community-acquired methicillin resistant Staphylococcus aureus in primary and high school students. Mikrobiyol Bul. 2008;42(4):661-7.

\section{Submit your next manuscript to BioMed Central and we will help you at every step:}

- We accept pre-submission inquiries

- Our selector tool helps you to find the most relevant journal

- We provide round the clock customer support

- Convenient online submission

- Thorough peer review

- Inclusion in PubMed and all major indexing services

- Maximum visibility for your research

Submit your manuscript at www.biomedcentral.com/submit
O Biomed Central 